\title{
A Knowledge Base System for Rapid Design of Harvesting Machinery Chassis
}

\author{
Yu Chen ${ }^{1, a}$, Yuefeng $\mathrm{Du}^{1}$, Zhenghe Song ${ }^{1}$, Zhongxiang Zhu ${ }^{1, \mathrm{~b} *}$ \\ ${ }^{1}$ Beijing Key Laboratory of Optimized Design for Modern Agricultural Equipment, College of \\ Engineering, China Agricultural University, Beijing, 100083, China \\ aemail: chenyu19890410@163.com, ${ }^{\text {be }}$ email: zhuzhonxiang@cau.edu.cn
}

Keywords: Knowledge Base; Harvesting Machinery Chassis; Rapid Design

\begin{abstract}
During the design of harvesting machinery chassis, a lot of factors should be considered, such as the standard data, expert experience, design formulas and the components' 3D models. It is very difficult to use and manage so many kinds of design data reasonably. Accordingly, the knowledge base system for design of combine harvester chassis is developed based on KBE in this paper. Based on the comprehensive analysis of structural characteristics and design requirements about harvesting machinery chassis, the design knowledge is divided into two parts as follows: one is case knowledge including machine parts, standard parts and hydraulic components; the other is rule knowledge composed of formulas, parameters and type selection. In consideration of the obvious structure of case knowledge and clear causal relationship of rule knowledge, an objectoriented hybrid knowledge representation method combining production knowledge representation with frame knowledge representation is proposed. According to the knowledge representation method, the attribute labels of structural parameters, performance parameters and concept description are determined, and the storage structures of assembly relationships and 3D models of instance knowledge are designed. Meanwhile, the knowledge tree approach is used to design the storage structure to save the rule knowledge such as formula sheets, parameter tables, rule tables, etc. Then the storage of knowledge is realized with the application of relational database SQL Server 2008. Finally, by using Visual Studio 2008 and ADO, a knowledge base system for rapid design of harvesting machinery chassis is built up. The function modules of user management, casebase management and rule-base management are designed and the user operation functions including the addition, modification, inquiry and deletion of knowledge are developed in this system. Most important of all, the system, which is not only convenient to use and manage the knowledge, but also comprehensive to support the rapid design of harvesting machinery chassis, will shorten the developing time of new products and improve design efficiency.
\end{abstract}

\section{Introduction}

Chassis is an important part of the vehicle. Different design methods for harvesting machinery chassis can directly affect the vehicle performance and the level of agricultural mechanization. The traditional design method has to take complex design knowledge, such as design manual, national standards and literature, into consideration in the design process. Moreover, the designed example knowledge has not been effectively utilized. Thus, the design cycle of traditional design is long and the efficiency becomes low, which lead to lack of market competitiveness [1].

Applying modern design techniques such as digital product design and rapid design method, some agricultural enterprises can solve the problems such as a lot of repetitive design and low utilization of information resource [2-6]. Nowadays, knowledge base system has been effectively utilized and developed in a number of industries both at home and abroad. A knowledge base system can avoid designers' large knowledge collection at the initial stage of the design work [7-9], which will effectively improve the management and use of knowledge and enhance the ability to design harvesting machinery chassis [10][11]. In this paper, a knowledge base system for rapid design of harvesting machinery chassis is built up. 


\section{System Architecture}

The knowledge base system for rapid design of harvesting machinery chassis is consisted of two parts: knowledge base and knowledge management system. The knowledge base storages some design-related knowledge, such as case knowledge and rule knowledge. As a core part of the knowledge base system, the knowledge management system provides a method to organize and manage the knowledge of knowledge base. According to the characteristics of harvesting machinery chassis knowledge, architecture of the knowledge base system is proposed as shown in Figure 1.

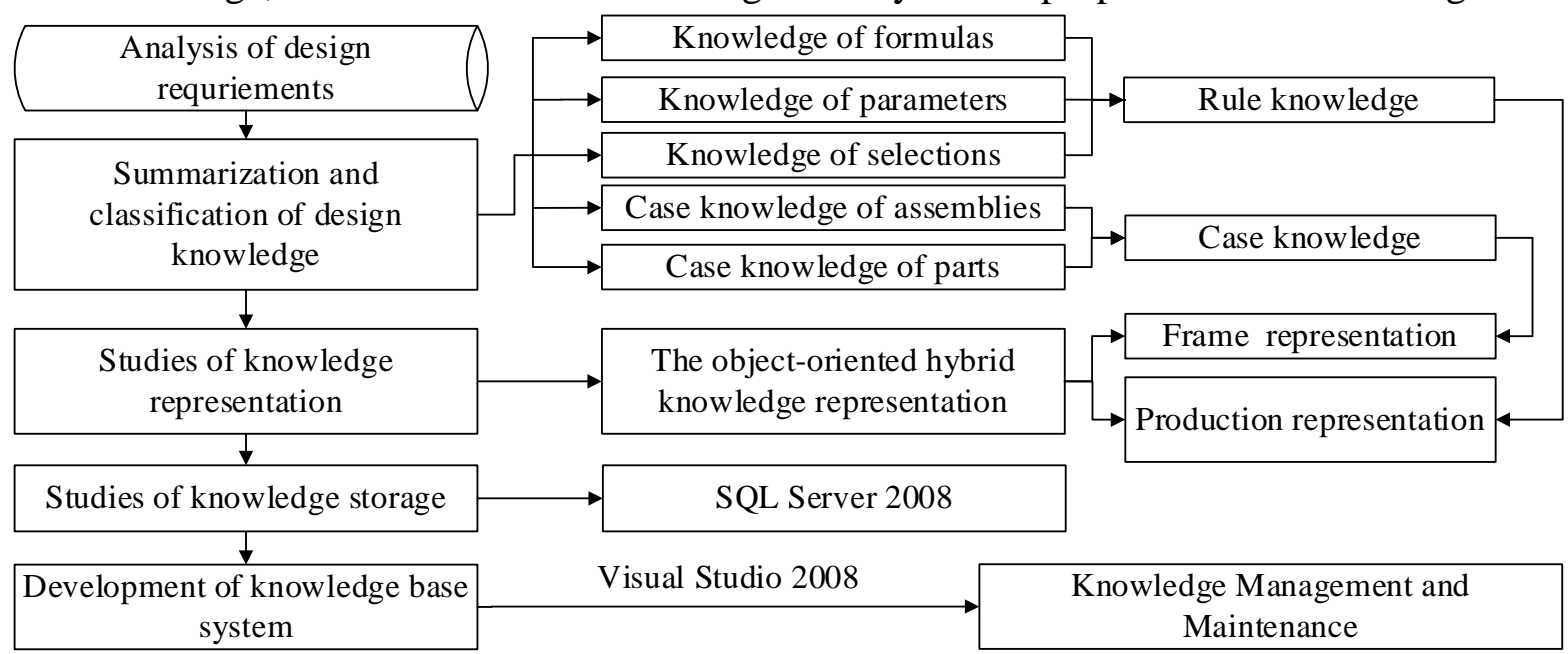

Fig.1. Architecture of the knowledge base system for rapid design of harvesting machinery chassis

The building process for knowledge base system of harvesting machinery chassis is listed as follows:

Setp 1: On the basis of analyzing design process of harvesting machinery chassis, the design knowledge is divided into case knowledge and rule knowledge, the case knowledge is consisted of systems, components and parts. The rule knowledge includes some design rules, such as parameters, formulas, charts, and so on.

Setp 2: The object-oriented hybrid knowledge representation which combining production representation with frame representation is adopted to express the rule knowledge and the case knowledge.

Setp 3: In accordance with the knowledge expression in Setp 2, the method of knowledge storage is determined and relational database SQL Server 2008 is selected to store all kinds of knowledge.

Setp 4: Visual C++ 2008 is applied to develop the knowledge base system interface, and ADO database development technology is used to access SQL Server 2008.

\section{Knowledge Representation}

Due to the complex structures and numerous species of harvesting machinery chassis, the domain knowledge which meets design requirements has a wide range of sources. In order to transform the domain knowledge into parameters which have a certain structure or sequential relationship, meet the requirements of inference rules and facilitate storage and management, it is need to take some specific methods to represent different types of knowledge.

It is depending on the knowledge of structural characteristics and organizational policies to choose method to represent knowledge [12]. According to the features of harvesting machinery chassis, the method of object-oriented hybrid knowledge representation which combining production representation with frame representation is used to express design knowledge. As the case knowledge has obvious structure and rule knowledge has clearly causality, the knowledge representation method of frame and production are respectively adopted to express the knowledge. Since the values of parameters change with the variant conditions, improved production is used to express dynamic parameters of the formula knowledge [13]. The classification and expression of harvesting machinery chassis knowledge is shown in Figure 2. Rule knowledge consists formulas, parameters and charts. Charts should be converted into mathematical model, such as functions or a 
set of discrete points, by mathematical methods. Case knowledge includes systems and components examples, as well as hydraulic and electrical components.

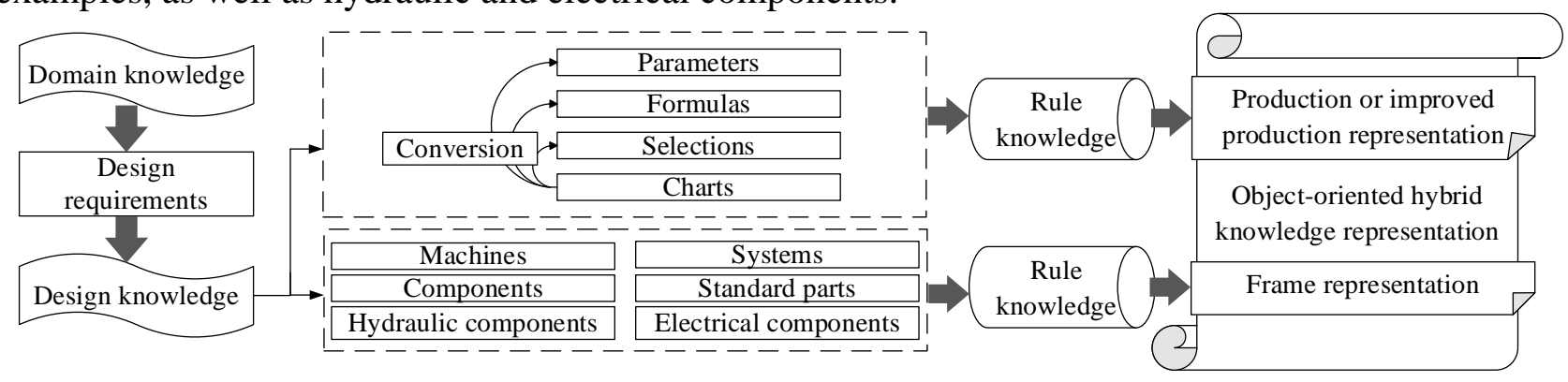

Fig.2. Classification and expression of knowledge

\section{Knowledge Storage}

Knowledge storage method should be able to meet the requirements of modularity and standardization to facilitate efficient and effective knowledge inquiry and management. In order to facilitate knowledge retrieval, encoding rules applicable to parts of harvesting machinery chassis are drafted according to the principles of uniqueness, invariance and scalability [14].

Case knowledge is modularized according to product ER model diagram. The storage method of the assembly relation is determined based on every contact properties in the model diagram. Then the instance is determined on the basis of each entity and its attributes in the model diagram.

The storage procedure of rule knowledge is shown in Figure 3. Firstly, the rule knowledge is modularized, and then the tree frame is selected to store the rule knowledge.

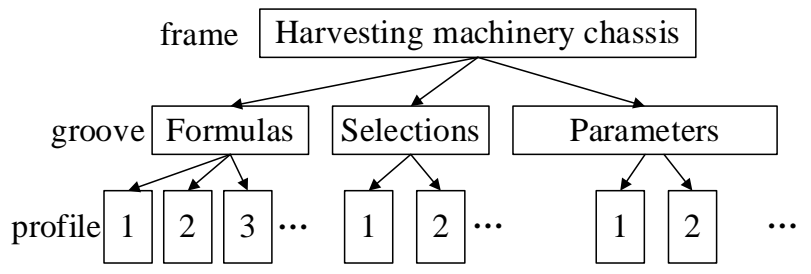

Fig.3. Storage procedure of rule knowledge

The structure of storage data is shown in Figure 4. According to parts ID, the various types of rule knowledge and case knowledge are associated with each other, which facilitates to achieve retrieval and use of the knowledge.

\begin{tabular}{|c|c|c|}
\hline Parameter table & Encode table & Formula table \\
\hline Subordinate ID & ID & Subordinate ID \\
\hline Name of parameter & Name & Name of formula \\
\hline Value of parameter & Degree & Content of formula \\
\hline Parameter condition 1 & Explanation & Parameter 1 \\
\hline$\ldots$ & Links of case & ... \\
\hline Parameter condition $n$ & & Parameter $\mathrm{n}$ \\
\hline Source of knowledge & Case table & Source of knowledge \\
\hline Confidence coefficient & $\ldots$ & Confidence coefficient \\
\hline
\end{tabular}

Fig.4. Structure of storage data

\section{System Implementation}

The main interface of knowledge base system includes rule library and case library management, which achieve navigation and management of the rule knowledge and case knowledge respectively. The menu bar of the system includes user registration, user login, system exits, user management, coding management, data backup, data batch import, data export and help documentation, etc.

The management interface of rule library is shown in Figure 5. When the tree control node of the "knowledge source" is clicked, corresponding original design data for referencing will be shown in pdf file format. When the tree control node of the "knowledge base" is clicked, formula tables, parameter tables and rule tables will be displayed in the list control on the right side of the interface. 
In Figure 5, the knowledge tables of belt are shown when the knowledge node "Belt transmission device" is clicked. The representational knowledge is displayed in the edit boxes when the project of the knowledge tables is clicked.

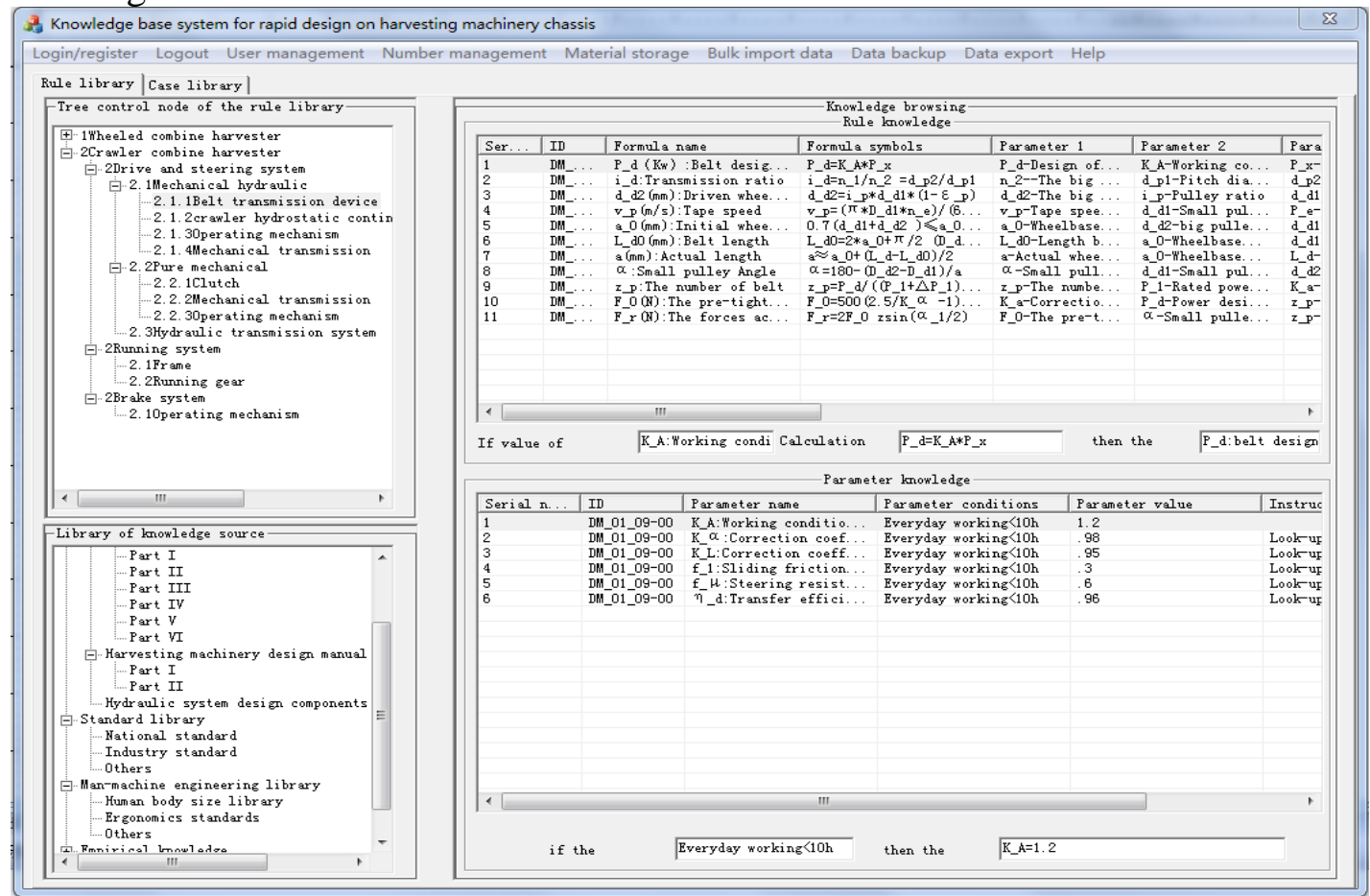

Fig.5. Management interface of rule library

The management interface of case library is shown in Figure 6. Assembly relationships, case tables and 3D models will be displayed on the right side of the interface when the tree control is clicked. In Figure 6, the information is shown when the node "crawler hydrostatic continuously variable transmission HST" of tree control is clicked.

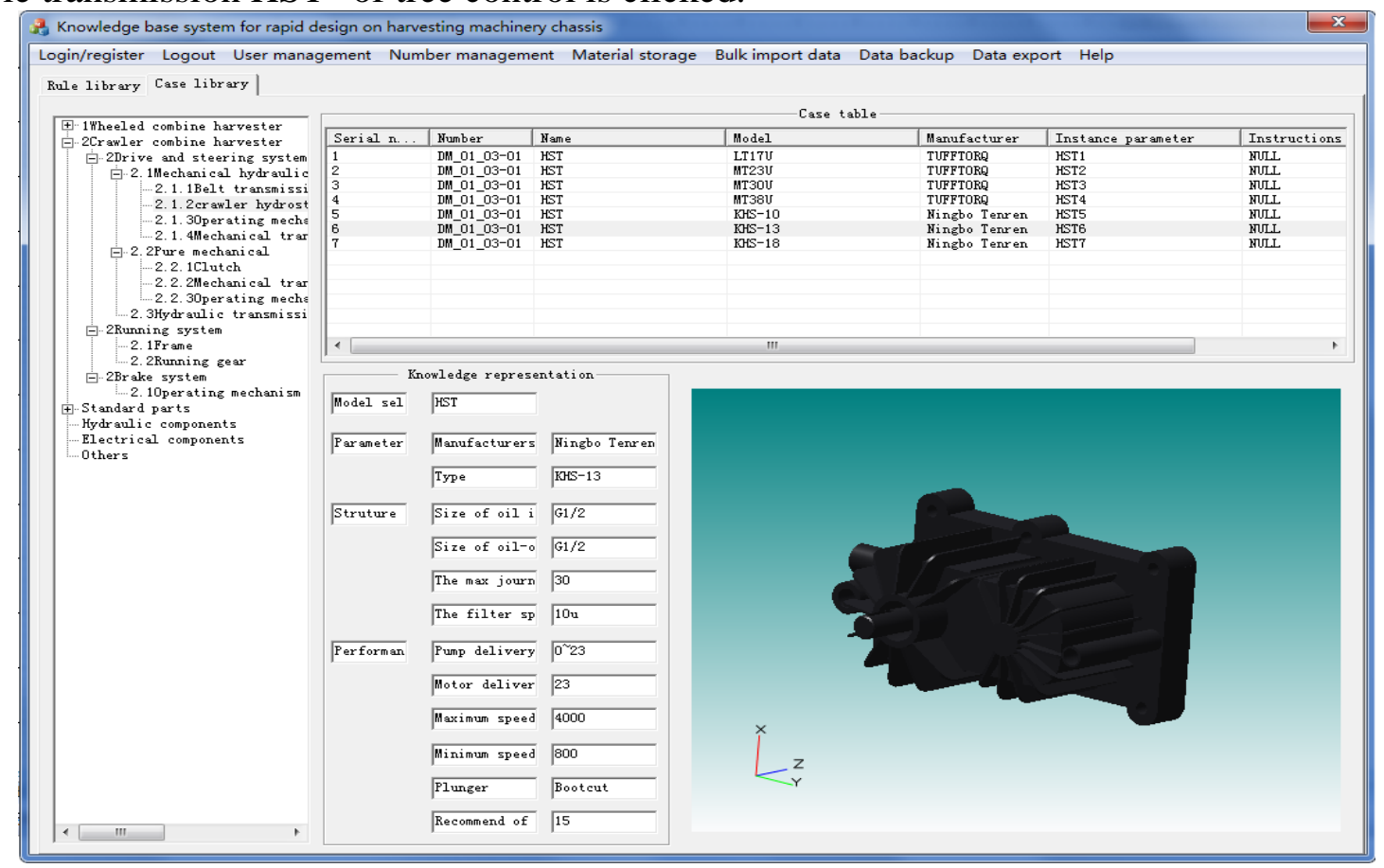

Fig.6. Management interface of case library

\section{Conclusions}

(1) According to harvesting machinery chassis design features, the knowledge was divided into case knowledge and rule knowledge. The object-oriented hybrid knowledge representation which 
combining production representation with frame representation was proposed to express rule knowledge and case knowledge.

(2) The method to store knowledge was determined in accordance with the characteristics of various types of knowledge. The knowledge management and maintenance methods designed were by analyzing the relationship among the various storage tables.

(3) Using ADO database access technology, combination development platform Visual C++ 2008 and data storage tools SQL Server 2008, the knowledge base system for rapid design of harvesting machinery chassis was developed.

The developed knowledge base system is not only easily to manage design knowledge, but also effectively to improve the ability of knowledge reuse to achieve rapid design of harvesting machinery chassis.

\section{Acknowledgements}

The research work was supported by the "12th Five-Year" National Sci-Tech Support Plan Project (2012BAF07B01) and Chinese Universities Scientific Fund (2014JD035).

\section{References}

[1] Wang Shucai, Dend Zaijing, Wen Youxian. Development strategies of agricultural machinery CAD in China [J]. Transactions of the Chinese Society of Agricultural Machinery, 2004, 01: 159$161,169$.

[2] Bi Shuqin, Song Zhenghe, Jin Xiaoping, et al. Design of knowledge base for crawler combine harvester transmission system [J]. Agricultural Engineering, 2013, 03: 94-98.

[3] Song Zhenghe, Bi Shuqin, Jin Xiaoping, et al. Rapid design reasoning method for crawler harvester transmission system [J]. Transactions of the Chinese Society of Agricultural Machinery, 2013, S2: 268-272.

[4] Li Changlin, Wang Xueliang, Jin Xiaoping, et al. Expert system design of chassis rapid design for high-speed transplanter $[\mathrm{J}]$. Transactions of the Chinese Society for Agricultural Machinery, 2012, 43(Supp): 323-328.

[5] Chen Yu, Mao Enrong, Lv Huangzhen, et al. Parametric design of large harvesting machinery transmission gearbox based reasoning [J]. Transactions of the Chinese Society for Agricultural Machinery, 2013, S2: 278-282.

[6] Chapman C.B., Pinfold M. Design engineering-a need to rethink the solution using knowledge based engineering [J]. Knowledge-Based Systems, 1999, 12: 257-267.

[7] Kumar S., Singh R. A low cost knowledge base system framework for progressive die design [J]. Journal of Materials Processing Technology, 2004, 153-154: 958-964.

[8] Naranje V., Kumar S. A knowledge based system for automated design of deep drawing die for axisymmetric parts [J]. Expert Systems with Applications, 2014, 41(4): 1419-1431.

[9] Fang Yonglong, Zou Naiwei, Yang Zhihua, et al. I/O interface design of auto brake expert system and construction of its knowledge base $[\mathrm{J}]$. Transactions of the Chinese Society for Agricultural Machinery, 2006, 06: 23-26.

[10]Ding Zhidong, Zheng Songlin, Shao Chen, et al. Configuration of knowledge base for auto digital development [J]. Journal of University of Shanghai for Science and Technology, 2005, 01: 91-94.

[11] Zhang Junyuan, Huang Jinling, Zhuang Weimin. Development methodologies for knowledge based design system [J]. Transactions of the Chinese Society of Agricultural Machinery, 2002, 06: 
110-112.

[12] Gu Lanzhi, Fang Yixiang. Design knowledge representation for product of valve and the design of knowledge base [J]. Journal of Hebei Polytechnic University (Natural Science Edition), 2009, 04: 47-50.

[13] Nian Zhigang, Liang Shi, Ma Fanglan, et.al. Study and application of knowledge expression [J]. Application Research of Computers, 2007, 05: 234-236, 286.

[14] Xie Qingsheng, Fu Xiang, Li Shaobo. Design and realization of encoding system for lowvolume but more-varieties products and parts in PDM system [J]. Journal of Computer Applications, 2009, S1: 356-358, 361. 